\title{
Prolonged Pain Relief With Use Of 10\% Lidocaine Neurolysis For Refractory Meralgia Paresthetica
}

\author{
Gaurav Chauhan, MD, Sanchit Ahuja, MD, David D Kim, MD, and Aman Upadhyay, MD
}

\section{ABSTRACT}

Meralgia paresthetica is a sensory neuropathy characterized by anterolateral thigh pain associated with paresthesiae. It is hypothesized that entrapment, compression, or stretching of the lateral femoral cutaneous nerve as it exits through the inguinal ligament. Often times life style modification, neuropathic pain medications, and or steroid injections can help relieve the pain. In some circumstances, the pain is refractory and more invasive procedures such as radiofrequen- cy ablation and or even surgery may need to be pursued. The authors report the successful use of $10 \%$ lidocaine for chemical neurolysis for Meralgia paresthetica in a 47-year-old female refractory to conventional treatment. In this case report, we will discuss the risk factors, pathomechanics, diagnostic challenges, therapeutic options and novel approach employed by the authors.

Key words: $10 \%$ lidocaine, chemical neurolysis, meralgia, aresthetica, neuropathic pain
Meralgia paresthetica (MP) is an entrapment mononeuropathy of the lateral femoral cutaneous nerve (LFCN); it is characterized by sensory symptoms in the anterolateral aspect of the thigh. It is a rare disorder with a reported incidence of 1 in 10,000 patients (1). The authors report the successful use of $10 \%$ lidocaine for chemical neurolysis in refractory MP in a 47-year-old woman who consented for this case report to be published. In this case report, we discuss the risk factors, pathomechanics, diagnostic challenges, therapeutic options, and novel approach employed by the authors.

\section{CLINICAL VIGNETTE}

\section{A 47-year-old woman with a body mass index (BMI)}

From: Henry Ford Health System, Detroit, MI

Author for correspondence: Gaurav Chauhan, MD

Mailing address: Department of Anesthesiology, Henry Ford Health System, CFP-342, 2799 W. Gr Blvd., Detroit, MI 48202

E-mail: Gchauha1@hfhs.org of $36 \mathrm{~kg} / \mathrm{m}^{2}$, total body weight of $107 \mathrm{~kg}$, and an established diagnosis of MP, morbid obesity, hypertension, and diabetes mellitus type II (well controlled) presented to our pain clinic with complaints of burning pain in her left thigh for the last 3 years. She reported that her pain was insidious in onset and had a waxing and waning course, with intensity ranging from 4 to 8 out of 10 on the Numeric Rating Scale (NRS-11) of pain; pain was exacerbated by walking, sitting for prolonged periods of time, or wearing tight clothes, and was rarely alleviated by lying down in a right lateral position. She reported that conservative management with opioids, nonsteroidal anti-inflammatory drugs, and gabapentinoids was suboptimal in controlling her pain. Her current analgesic regimen included 90 $\mathrm{mg}$ of pregabalin 3 times a day, $80 \mathrm{mg}$ of topiramate twice a day, $750 \mathrm{mg}$ of methocarbamol 3 times a day, $50 \mathrm{mg}$ of diclofenac twice a day, along with $25 \mathrm{mg}$ of nortriptyline nightly. The patient further reported that she underwent both pulsed and continuous radiofrequency ablation (RFA) of the LFCN on separate occasions, with pain relief not lasting more than 6 weeks. The patient stated that after multiple cycles of therapeutic failures, she was getting increasingly frustrated and depressed due to her chronic pain. She 


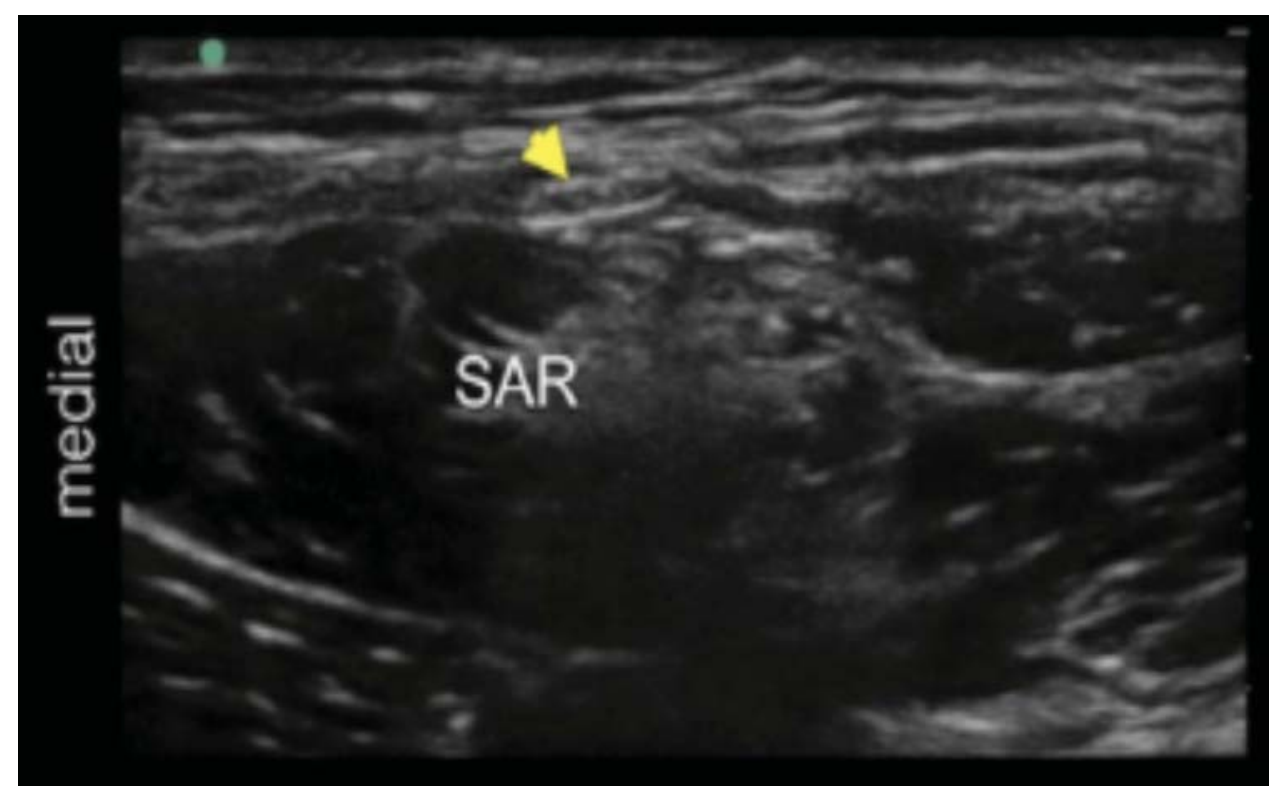

Fig. 1. USG image of lateral femoral cutaneous nerve (yellow arrowhead) approximately 3 finger breaths caudal to anterior superior illiac spine (ASIS) and cephalad to sartorius (SAR). .

revealed that the pain was now adversely affecting her quality of life and was beginning to restrict her daily activities.

The patient was deemed a candidate for chemical neurolysis with $10 \%$ lidocaine solution. The risks, benefits of the procedure, and other therapeutic alternatives such as nerve decompression surgery were explained in detail to the patient. She preferred chemical neurolysis and subsequently consented to the procedure. On the day of the procedure, the patient was placed in the supine position and procedural sedation was instituted with $2 \mathrm{mg}$ of midazolam intravenously. Under ultrasound guidance (USG) and sterile precautions, the USG probe was placed immediately below the anterior superior iliac spine (ASIS), parallel to the inguinal ligament. The sartorius muscle was identified and followed laterally until the fascia containing the nerve was well visualized (Fig. 1). The point of injection was $2 \mathrm{~cm}$ medial and $0.5 \mathrm{~cm}$ inferior to the ASIS. A 22-gauge needle was advanced at that point, between the fascia lata and the sartorius muscle (Fig. 2). After negative aspiration for blood, $3 \mathrm{~mL}$ of a solution containing $10 \%$ lidocaine and $40 \mathrm{mg}$ of methylprednisolone acetate (Depo Medrol) was injected (Fig. 3).
The patient tolerated the chemical neurolysis with $10 \%$ lidocaine well. No side effects were reported by the patient or observed by the authors. The patient reported excellent pain relief with remission of symptoms at the 1-month and 3-month follow-up appointments. At her 6-month follow-up appointment, she did report that her pain and associated symptoms had returned, albeit with lower intensity; these symptoms were optimally controlled with the current pain medication prescribed to her. The patient requested to schedule repeat neurolysis with $10 \%$ lidocaine under USG and for nortriptyline to be tapered off.

\section{DISCUSSION}

The pathomechanics of MP involves neuropraxia or permanent injury due to compression of the LFCN, as it courses along the posterolateral aspect of the psoas major muscle, crossing between the ASIS and the inguinal ligament before entering the thigh (2). The nerve is usually located 1 to $2 \mathrm{~cm}$ medial and inferior to the ASIS and 0.5 to $1 \mathrm{~cm}$ deep from the skin. However, the physician should be cognizant of the fact that upon entering the thigh, the LFCN divides into multiple branches innervating the lateral 
and anterior parts of the thigh. The variable anatomy poses a challenge during the conduct of a nerve block, using the landmark-based technique. This can be circumvented by using a USG approach (2). The USG probe should be placed immediately inferior and parallel to the inguinal ligament. The operator should use the lateral edge of the sartorius as a landmark, as the LFCN usually runs from the lateral to the medial edge of the superficial fascia of the sartorius muscle. The nerve is usually visualized as an oval hypoechoic structure with a hyperechoic rim of connective tissue, between the fascial lata and sartorius muscles (2).

Several metabolic and mechanical factors increase the risk for developing MP, such as obesity, diabetes, history of previous spine surgeries, procedures involving the ASIS, total hip replacement, lateral positioning during surgery, use of pelvic bolsters, etc. $(3,4)$. The pathophysiology of entrapment is complex, commencing with disturbances in neuronal microcirculation and vascular permeability leading to neuronal edema and inflammation (5). These responses provoke a cascade of events that induce neural plasticity, which entails recruitment of subthreshold synapses to nociceptive neurons, leading to sensitization and augmented response to normal stimuli (6). These maladaptive changes at the cellular and molecular levels manifest systemically as neuropathic pain, hyperreflexia, and dystonia $(6,7)$.

The symptoms of MP range from tingling and numbness along with burning pain in or on the lateral part of the thigh to dull pain in the groin area or across the buttocks, and may intensify after walking or standing. The

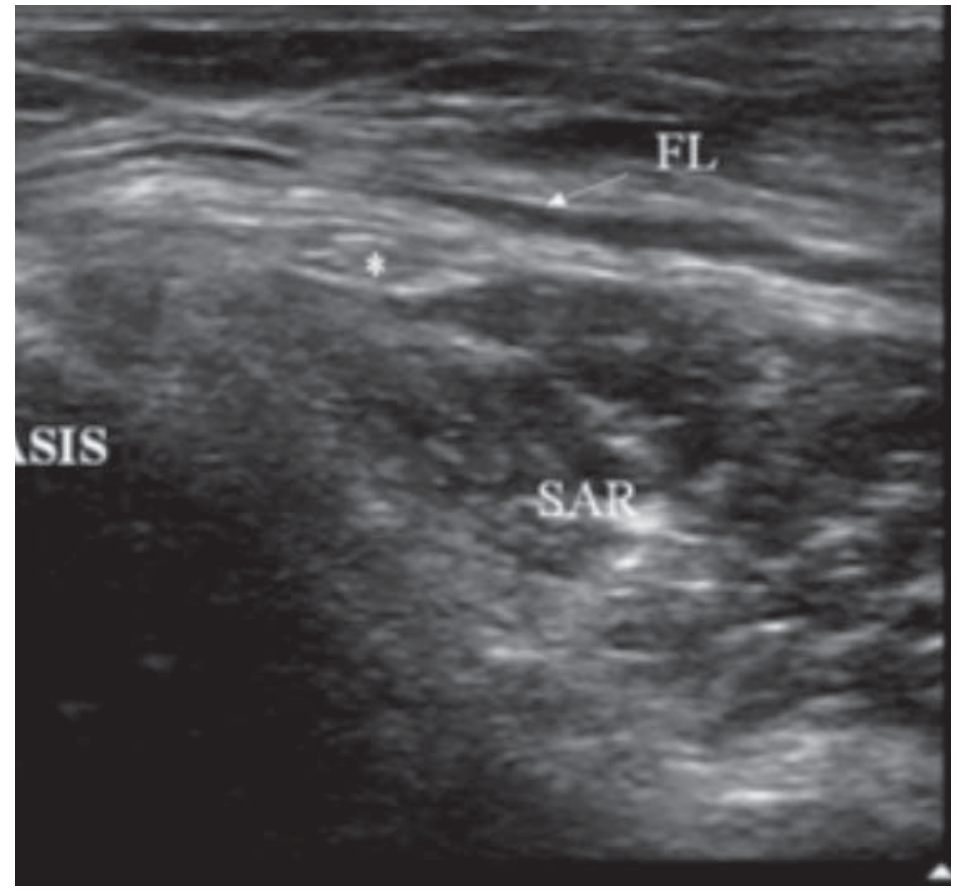

Fig. 2. USG image of lateral femoral cutaneous nerve (white star) before injection. ASIS = anterior superior iliac spine; FL: fascia lata; SAR: sartorius..

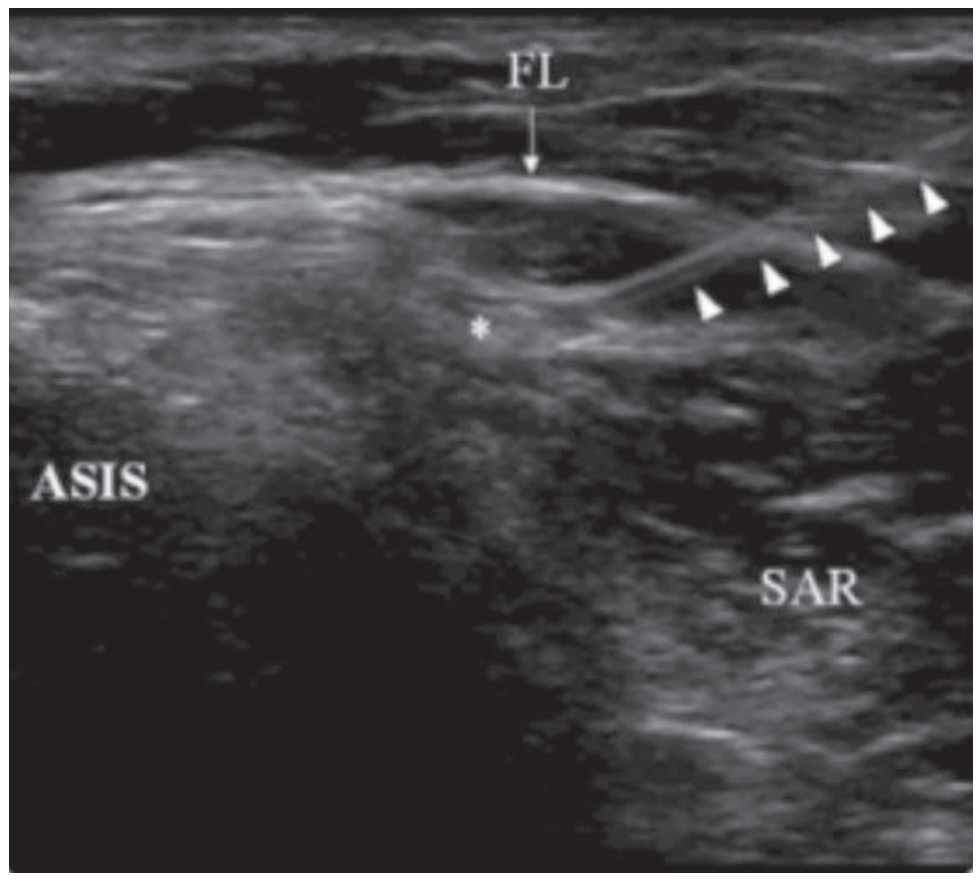

Fig. 3. USG image of lateral femoral cutaneous nerve (white star) after injection. ASIS = anterior superior iliac spine; FL: fascia lata; SAR: sartorius.. 
diagnosis of MP is clinched by the pathognomonic signs and symptoms and after an exhaustive clinical and neurological examination excludes any neurological complication due to regional anesthesia or surgery performed (8). Conservative measures like weight loss, physiotherapy, avoidance of external compression, and analgesic medications are successful in more than half of cases and are the mainstay of treatment. However, as a second-line therapy or as an adjunct to the current analgesic regimen for MP, perineural injection of a local anesthetic with or without corticosteroids at the presumed site of entrapment has been reported to be clinically successful (8). However, few patients are refractory to these measures and may need more invasive procedures like RFA or even nerve decompression surgery. Although RFA is considered safer than surgical decompression, it may prove to be technically challenging due to the small segment of nerve involved, and results could be operator-dependent (9). To date, no clinical studies have yielded concrete evidence of the effectiveness of RFA for MP $(8,9)$. Furthermore, nerve decompression surgery is reported to have a success rate ranging between $50 \%$ to $90 \%$ and is usually more beneficial when the underlying etiology is external compression of the nerve. Consequently, current literature yields equivocal evidence to recommend either RFA or surgical nerve decompression (8).

Neurolytic agents such as phenol and alcohol can also be employed for neurolysis and the management of chronic pain. However, these agents are not specific in targeting neural tissue and may damage the surrounding tissues, with potential to cause cosmetic disfigurement. Lidocaine, an amide local anesthetic (LA), inhibits fast sodium channels on the axonal membrane of nerve, interrupting the transmission of impulses leading to a decrease in spontaneous and triggered after-discharges and a reduction in crossexcitation among neighboring afferent nerve fibers (10). The relationship between a higher concentration of lidocaine and volume is believed to be the main determinant of the duration of the therapeutic blockade (11). Ready et al used varying concentrations of lidocaine, ranging from $2 \%$ to $32 \%$, in the intrathecal space and reported cellular changes consistent with neurolysis starting at $8 \%$ or higher concentrations of lidocaine (12). Kim et al reported that $10 \%$ lidocaine, in vivo, yields histologic evidence of neurolysis, such as loss of gross architecture, endoneural edema, axonotmesis, etc. (13). Han et al used $10 \%$ lidocaine in patients with trigeminal neuralgia and concluded that, along with the relative simplicity inherent to the procedure, chemical neurolysis with $10 \%$ lidocaine is safe to perform, associated with complete reversibility of induced sensory deficits, and yields a longer duration of pain relief comparable to that with RFA (14). The authors, based on the facts mentioned above and their previous experience with the successful use of $10 \%$ lidocaine in other refractory chronic pain states, decided to employ $10 \%$ lidocaine as their agent of choice for neurolysis. The side effects reported with the higher concentration of lidocaine are a risk of toxicity that may manifest as transient neurologic symptoms, with an incidence of $4 \%$ to $33 \%$, as per current literature. Other adverse effects such as dizziness, blurred vision, profound hypertension, slurred speech, pseudoseizures, and seizures range from $0.1 \%$ to $9 \%$ and are proposed to be independent of the concentration or dose of the lidocaine used (15).

\section{CONCLUSION}

This case report yields evidence that chemical neurolysis with $10 \%$ lidocaine yields optimum symptom control for up to 6 to 7 months in cases with refractory MP, and may be considered as an alternative to other therapeutic modalities such as RFA in cases of MP refractory to conventional treatment. Alleviation of pain to tolerable limits interrupted the vicious cycle of chronic pain and its psychological sequelae. Furthermore, with reversibility of induced sensory functional deficits, chemical neurolysis with $10 \%$ lidocaine offers an advantage over RFA. Chemical neurolysis may also be best-suited for patients who are considered to be high-risk for nerve decompression surgery due to significant comorbidities. However, careful selection of patients is vital, as MP may mimic or coexist with low back pain and other radicular symptoms. Hence, establishing the cause of pain as localized and nerve-specific, and ruling out any psychological components, are essential before proceeding with any interventional pain procedure. The authors' conclusions are limited as they are based on the clinical outcome of a single patient, and a case series or a randomized clinical trial should be designed to verify the reported observations. 


\section{REFERENCES}

1. Latinovic R, Gulliford MC, Hughes RA. Incidence of common compressive neuropathies in primary care. J Neurol Neurosurg Psychiatry 2006; 77:263-265.

2. Dias FLC, Valença MM, Guimaräes Filho FA, Medeiros RC, Silva RA, Morais MG, Valente FP, Franca SM. Lateral femoral cutaneous neuralgia: An anatomical insight. Clin Anat 2003; 16:309-316.

3. Chauhan G, Gupta K, Nayar P. Meralgia paresthetica after total hip arthroplasty in supine position. Saudi J Anaesth 2013; 7:105-106.

4. Gupta A, Muzumdar D, Ramani PS. Meralgia paraesthetica following lumbar spine surgery: A study in 110 consecutive surgically treated cases. Neurol India 2004; 52:64-66.

5. Navarro X, Vivó M, Valero-Cabré A. Neural plasticity after peripheral nerve injury and regeneration. Prog Neurobiol 2007; 82:163-201.

6. Woolf CJ. Central sensitization: Implications for the diagnosis and treatment of pain. Pain 2011; 152:S2-S15.

7. Onat, SS, Ata AM, Ozcakar L. Ultrasound-guided diagnosis and treatment of meralgia paresthetica. Pain Physician 2016; 19: E667-E669.

8. Khalil N, Nicotra A, Rakowicz W. Treatment for meralgia paraesthetica. Cochrane Database of Systematic Reviews 20012; 3:CD0004159.
9. Philip CN, Candido KD, Joseph NJ, Crystal GJ. Successful treatment of meralgia paresthetica with pulsed radiofrequency of the lateral femoral cutaneous nerve. Pain Physician 2009; 12:881-885

10. Lavoie PA, Khazen T, Filion PR. Mechanisms of the inhibition of fast axonal transport by local anesthetics. Neuropharmacology 1989; 28:175-181.

11. Nakamura T, Poppitz-Bergez F, Birknes J, Strichartz GR. The critical role of concentration for lidocaine block of peripheral nerve in vivo: Studies of function and drug uptake in the rat. Anesthesiology 2003; 99:1189-1197.

12. Ready LB, Plumer MH, Haschke RH, Austin E, Sumi SM. Neurotoxicity of intrathecal local anesthetics in rabbits. Anesthesiology 1985; 63:364-370.

13. Kim DD, Asif A, Kataria S. Presentation of neurolytic effect of $10 \%$ lidocaine after perineural ultrasound guided injection of a canine sciatic nerve: A pilot study. Korean J Pain 2016; 29:158163.

14. Han KR, Kim C, Chae YJ, Kim DW. Efficacy and safety of high concentration lidocaine for trigeminal nerve block in patients with trigeminal neuralgia. Int J Clin Pract 2008; 62:248-254.

15. Yang S, Abrahams MS, Hurn PD, Grafe MR, Kirsch J. Local anesthetic Schwann cell toxicity is time and concentration dependent. Reg Anesth Pain Med 2011; 36:444-451. 